\title{
LISTADO DE EVALUADORES
}

Los estudios y artículos recibidos en CLARIDADES. REVISTA DE FILOSOFÍA son sometidos a un proceso de arbitraje ciego por dos informantes externos, los cuales se ocupan de evaluar el contenido y la metodología de los artículos. Todos los artículos tienen una revisión previa por parte del equipo directivo de la revista, para comprobar que los artículos cumplen los requisitos mínimos. Los autores reciben un resumen de los informes redactados por los revisores, indicándoles -si así es el caso- la manera de subsanar deficiencias o realizar los cambios que se solicitan. Los informantes son seleccionados de acuerdo a un criterio de excelencia académica e investigadora, y tomando en consideración que su ámbito de especialización se corresponda con las temáticas abordadas en cada uno de los artículos. En este número de la revista han colaborado como informantes las siguientes personas:

Alberto Ciria (Instituto Lauth, Alemania)

Alejandro Rojas (FICUM, España)

Antonio de Diego González (Universidad de Sevilla, España)

Antonio Miguel López Molina (Universidad Complutense, Madrid)

Claudia Fernández (Universdiad de Málaga, España)

Daniel Boxó (IES Nicolás Copérnico, España)

Davide E. Datturi (Universidad Autónoma, México)

Juan A. García González (FICUM, España)

Lucas Risoto (Universidad de Málaga, España)

Luis Felip López-Espinosa (IES Cerro del viento, España)

M. Parmeggiani (Universidad de Málaga, España)

Ma Teresa González Astudillo (Universidad de Salamanca, España) 
Paloma García (Colegio San José de Estepona, España)

Raquel Guerrero (Universidad de Málaga, España)

Raúl Caballero (Universidad de Málaga, España)

Sylvia Rodríguez (Universidad Autónoma de Madrid, España)

Claridades. Revista de filosofía 9 (2017) 